$\mathbf{X X I}$.

\title{
Über den weichen und steifen (nervösen) Leib.
}

\author{
Von
}

\author{
Dr. BENDERSKI \\ in Kiow.
}

Bei der Untersuchung des Abdomens stossen wir auf zweierlei Erscheinungen. Einerseits haben wir einen normal-weichen I eib, dabei kann die Untersuchung des ganzen Abdomens leicht ausgeführt werden. Wenn aber diese Weichheit die mittleren Grenzen überschreitet, so muss sie als abnorm bezeichnet werden. Solche Leiber findet man bei Vielgebärenden, bei den Trïgern verschiedener Ptosen (Gastro-Enteroptose, herabgesenkte IJeber, wandernde Niere u. dergl.). Das gleiche bemerkt man bei Individuen, bei welchen die Mm. rect. abdominis gelockert und diastasiert sind (wobei NB. die Muskeln, die Recti selbst, zuweilen sehr gespannt und wie zwei näulen ${ }^{*}$ stehen) und bei Hernienträgern, wobei die Umbilikalhernie zuweilen so gross ist, dass man in die Öffnung mit der ganzen Hand hineingreifen kann. Der exquisit weiche Leib ist fast immer ein Privilegium der Frauen, man findet ihn selten auch bei alten Männern (bei Hernienträgern).

Andererseits treffen wir sehr steife, harte, gespannte Leiber. Die extremen Grade der Spannung, wenn diese Spannung lange und fast unaufhörlich anhält, wenn der Leib brett-, fast beinhart wird, findet sich gleichfalls öfter bei Frauen, als bei Männern. Es gibt aber auch Übergangsstadieu zwischen dem normalen und dem sehr „nervösen Leib“. Dieser nervöse Leib „mittleren“ Grades zieht sich zusammen, zuckt bei der Berülıung mit der untersuchenden Hand, die Muskeln nspielen" auf solchem Leibe schon aus Furcht vor der bevorstehendeu Untersuchung, solche Individuen haben Furcht vor "Kitzeln“. Solche mittlere nervöse Leiber trifft man oft auch bei Männern, auch bei jungen, kräftigen Männern, und ich würde in Verlegenbeit geraten, wenn man mich 
fragte, wer den quantitäten Vorzug hat. Qualitativ, wie gesagt, steben die Frauen an der ersten Linie.

Selbstverstiindlich spreche ich hier nicht von der Spannung des Leibes, welche mit exquisiter Fettanhäufung, Meteorismus, Ascites, Neoplasma im Zusammenhange steht. Die steifen Leiber müssen auch nicht gross sein, sie sind eher klein, flach zusammengezogen. Ich spreche vom nervösen Zustande, welcher sich in Form der Spannung der Bauchmuskulatur darstellt, ganz gleich, ob es eine periphere, reflektorische oder zentrale Erscheinung ist. Verschiedene Gruppen der Bauchmuskulatur sind gespannt, mehr aber sieht man es, wie mir scheint, an den Recti abdominis, und zwar mehr an ihrer oberen Hälfte, oder an den oberen zwei Dritteln derselben. Diese Muskeln sind fast immer gespannt, auch ohne dass man sie berührt, und bei der Untersuchung wird die Spannung noch grösser, auch wenn kein Temperaturunterschied zwischen der Haut des Patienten und den Fingern des Arztes vorliegt.

Es entstehen zwei Fragen: Welchen Schluss kann man aus diesen Zuständen - wollen wir der Kürze wegen sagen - aus der Härte und der Weichheit des Leibes, auf den allgemeinen Zustand des ganzen Organismus ziehen? Besonders wichtig erscheint tür mich die Frage, ob man aus diesen Zuständen keine Anhaltspunkte für die Diagnose der Krankiheiten des Magendarmkanals gewinnen kōnnte.

Die Träger des weichen Leibes sind oft auch im allgemeinen "weiche" atonische Leute, und dieser atonische Habitus stimmt vollkommen mit der Vorstellung ron der Ptose, als von einer konstitutionellen Krankheit. Bei solchen Individuen ist sehr leicht eine atonische - mechanische und chemische - Arbeit des Verdauungskanals denkbar. Symptomatisch leiden solche Leute an Obstipation, kaum seltener kommen bei ihnen auch Diarrhoen vor, öfter kommt bei ihnen die intermittierende Form, einmal Obstipation, einmal Diarrhoen, vor.

Die harten, nervösen Leiber sind charakteristischer. Bei den Trägern und Trägerinnen des nervösen Leibes, schwachen, mittleren und besonders starken Grades kommen immer Zeichen der allgemeinen Neurasthenie zum Vorschein.

Wenn ich die Spannung, die Zuckung, das "Spiel" nur der Bauchmuskulatur vor mir sehe, da sage ich mir, ich habe mit einem "nervösen" Individuum zu tun. Ich fange an, den ganzen Organismus zu untersuchen, nehme die Anamnese auf und finde meine Vermutung ausschliesslich positiv bestätigt. 
Benderski, Über den weichen und steifen (nervösen) Leib.

Der nervöse Leib dient mir als positiver Barometer zur Feststellung der nervōsen Balance des betreffenden Individuums.

Das ist allgemein verstiindlich und kann als wenig neu erscheinen. Ich lege mehr Gewicht auf die andere Seite des Schlusses, und zwar, ob es möglich ist, von dem nervösen Leibe einen Schluss oder eine Vermutung auf den Charakter der Erkrinkung des Magendarmkanals abzuleiten.

Ja, man kann das, und das hat eine beträchtliche praktische Bedeutung. Jederman weiss, wie spärlich wir quantitativ und qualitativ über die sogenannte nervöse Dyspepsie unterrichtet sind. Wir wissen nur, dass die Erkrankangen des Verdauungstractus nervöser Natur sehr zilhlreich und mannigfaltig sind, um so mehr, als die mechanischen und sekretorischen Funktionen der Verdaungsorgane im engen Zusammenhange mit dem Nervensystem stehen.

Es ist in jedem einzelnen Falle sehr wichtig, zu wissen, welcher Natur diese oder jene Krankheit ist. Obwohl mit der Bezeichnung nnervōse Erkrankung “ des Magens noch nicht viel gesagt ist, weiss doch jeder praktische Arzt, wie wichtig es doch für die Wahl der therapeutischen Mittel ist und welchen Einfluss es auf den Gang der Heilung haben kann. Ich messe deswegen dieser Frage auch schon in dieser Form eine beträchtliche Bedeutung bei.

Ich behaupte, dass man bei den Träigern des nervösen Leibes gewiss eine Reizung des allgemeinen Nervensystems annehmen darf, und wenn ein solches Individuum, was wichtiger ist, mit Klagen über Dyspepsie auftritt, so hat man Grund, anzunehmen, dass diese Defekte des Verdaungskanals auf nerröser Basis beruhen.

Dann wird der Ausgaugspunkt richtig Zeit und Gewinnst für den Arzt und für den Patienten.

Und freilich konnte ich in vielen Fïllen die Richtigkeit der gemachten Vorschriften verifizieren, inden die frühere Therapie, welche auf falschem Boden basiert war, keine positiven Resultate geben konnte.

Ich möchte auch auf einen Überga ngsustand die Aufmerksamkeit lenken. Es gibt Kranke, bei welchen der Leib weich ist, die I) armschlingen aber sind hier und da melır oder weniger zusammengezogen und gespannt, meistens halb gespannt. In solchen Fällen kann man das Ceocum, die Sigmoidea in Form von 
mehr oder weniger dilatierten, zusammengezogenen Würstchen palpieren. In solchen Füllen kann man auch zuweilen - durchaus nicht oft - das Col. transversum abtasten.

Es fragt sich, welche Grundsätze können wir alus den beschriebenen Zustïnden des Abdomens für die Therapie der Magendarmkrankheiten aufstellen? In sehr allgemeinen Umrissen gehe ich so vor:

Bei weichem Leibe empfehle ich ein allgemein tonisierendes und roborierendes Regime, für den Leib - bei passender Diït energische, stimulierende, gewöhnliche oder Vibrationsmassage. Wenn nötig, ein Gürtel auf den Leib. (Von spezifischen Indikationen sehe ich natürlich ab.) Beim steifen, nervösen Leib empfehle ich ein allgemein beruhigendes Regime bezw. Bäder, Kompresse auf den Leib. Am besten aber wirkt hier die Massage unter Wasser, verbunden mit einer warmen Douche auf den Leib; nach meiner Methoded ${ }^{1}$ ), wende ich zuweilen die Vibration unter Wasser an.

Ich teile also den Leib nach seinem Spannungszustande in verschiedene Kategorien:

Der normale Leib - die Bauchmuskulatur hat einen gewissen Tonus, der Leib bleibt aber weich, und man kann ihn leicht untersuchen.

Sehr weicher Leib - breiartiger Leib - in meinem Krankenbuche notiere ich solchen Leib kurz Leib-KaschaBreileib-Chiffon und der nervöse Leib, der steife Leib, der Brettleib.

Nach dem Zustande des Leibes kann man ein Urteil über den allgemeinen Zustand des Nervensystems und die nervöse Balance des Verdauungskanals fällen.

Besonders klar dient der nervöse Leib als Beweis für die allgemeine Nervenreizung und für den nervösen Charakterder Verdaungsleiden, was für die Therapie von Wichtigkeit ist.

Nehmen wir zum Beispiel das Sodbrennen, das Aufstossen, verschiedene Leibschmerzen. Es ist doch nicht immer leicht zu sagen, ob diese Symptome auf motorischer, sekretorischer oder gar diätetischer Grundlage fussen. Der nervöse Leib gibt uns oft leichten Aufschluss. Es gibt sehr hartnäckige nervöse Leiber. Ich behandle eine junge Dame schon seit 8 bis 9 Jahren. Sie hat einen echten Brettleib und leidet an periodischen,

1) Erscheint demnāchst in der Wiener med. Wochenschrift. 
Benderski, Über den weichen und steifen (nerrōsen) Leib. 269

heftigen Krampfanfällen des Leibes. Hier tritt sehr klar die Erweichung, der Bauchmuskulatur bei der Anwendung meines Verfahrens der Massage unter dem Wasser zutage. Die Krankheit rezidiviert aber. Übrigens ist es ein Unikum. Die Patientin hat sich der Krankheit wegen von dem Manne scheiden mũssen. Ihre Leiden sind ausserordentlich gross. Sie kommt zu mir aus der Provinz zweimal jährlich, ich mache die Schmerzen und die hartnaickigste Obstipation etwas leichter, sie verreist, dann rezidivieren die Anfälle wieder.

Aus dem Charakter der Krankheit allein geht hervor, dass sie hartnäckig und zu Rezidiven geneigt sein muss; ich behaupte aber noch einmal, dass der nervöse Leib, indem er Zeichen derallgemeinen Nervenreizung ist, als wichtiger Indikator der nervösen Balance des Magendarmkanals dienen und damit nützliche Hinweise für die Therapie geben kann. 